\title{
Mosaic in two colors
}

A new strategy for the creation of genetic mosaics by interchromosomal recombination in mice allows the tracking of single cells with recombinant genotype and their progeny through development.

Art historians and geneticists have at least one thing in common: they both get very excited about mosaics. While the former will revel in the beauty of an artwork made up of tiny pieces of glass, the latter will avidly study a multicellular organism composed of cells with different genetic background.

Liqun Luo and his team at Stanford University saw mosaics as the ideal way to follow the function of a particular gene in a biological process. They sought not only to create a mosaic of cells homozygous for a mutant gene against a background of heterozygous cells, but also to follow the progeny of these cells and to study the effect of the genetic alteration on development. "We wanted to knock out ubiquitously expressed genes in only a small population of neurons, not the whole animal," Luo explains, "and assay their loss-of-function phenotype in neuronal morphogenesis."

Initially they applied this idea to Drosophila melanogaster. The fly is cheap and easy to work with, but Luo also wanted to adapt their method to a mammalian system, and in a recent article in Cell, his team introduces a method to do mosaic analysis with double markers in mice (MADM).

The principle of MADM is straightforward: two mouse lines are generated, encoding either the $\mathrm{N}$ terminus of green fluorescent protein (GFP) and the $\mathrm{C}$ terminus of red fluorescent protein (RFP) split by a loxP site (termed GR), or the reverse, with the $\mathrm{N}$ terminus of RFP and C terminus of GFP (termed RG). The GR markers are positioned proximal to a mutation of interest, the RG markers proximal to the wild-type gene. In addition, the RG strain contains Cre recombinase, the enzyme necessary to induce recombination at the loxP site. Upon crossing of these two strains, Cre induces interchromosomal recombination; cells with a restored green fluorescent marker will be homozygous for the mutant, red cells will be homozygous for the wild-type gene, and all cells with double color retain their heterozygous phenotype (Fig. 1). All that is left to do is to follow the cells of different color to determine how

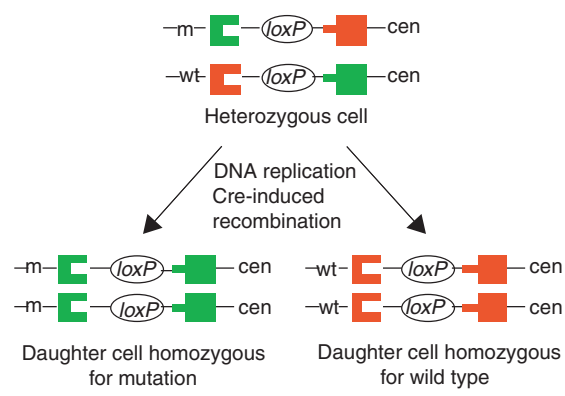

Figure 1 | Principle of MADM at the cellular level in a mosaic mouse. Upon interchromosomal recombination at the loxp site, which is located between the centromer (cen) and the mutated (m) or wild type (wt) gene of interest, cells are either homozygous for the green or red markers and all genes distal to them, or remain heterozygous and thus labeled with both markers.

each contributes to a phenotype of interest. Interchromosomal recombination only happens with very low frequency, but Luo sees this as advantageous in most cases. "You can study, with really high resolution, what happens to a single cell with a deleted gene, when all its neighbors are wild type."

There are many exciting applications for MADM, and one of the most interesting to Luo is its use in cancer research. "It could be very useful to study tumor progression in vivo," he points out. A tumor suppressor mutant and its wild-type counterpart would each carry the RG or GR markers, and upon recombination, cells homozygous for the mutant and all daughter cells will carry the green marker, allowing for easy tracking of their contribution to tumor formation.

At present the technique is restricted to genes located on the small arm of chromosome six, distal to the ROSA26 locus, a well characterized chromosomal position known to ubiquitously express any gene inserted there and therefore ideal for the insertion of the MADM markers. Luo is optimistic that with better characterization of the mouse genome, similar loci on other chromosomes will be found that will allow the expanded use of MADM as a tool to study the role of different genes in mouse development.

\section{Nicole Rusk}

\section{RESEARCH PAPERS}

Zong, H.et al. Mosaic analysis with double markers in mice. Cell 121, 479-492 (2005). 